Full Paper

\title{
On the Mechanism of Energy Transfer in the Plasma-Propellant Interaction
}

\author{
Andrew J. Porwitzky*, Michael Keidar, Iain D. Boyd \\ University of Michigan, Department of Aerospace Engineering, Ann Arbor, Michigan 48109-2140 (USA) \\ DOI: 10.1002/prep.200700042
}

\begin{abstract}
A coupled plasma sheath/ablation model is developed for electrothermal chemical gun applications. By combining a commonly employed collisional sheath model with a previous ablation model, the convective heat flux as a function of time to the propellant bed is determined for two potential electrothermal chemical gun propellants, XM39 and JA2. It is found that the convective heat flux varies smoothly from a nearly collisionless to a fully collisional regime over the short duration of the plasma pulse. The possibility of determining an accurate estimate of the amount of heat flux to the propellant bed due to radiation from the bulk plasma presents itself.
\end{abstract}

Keywords: ETC, Heat Flux, Modeling, Plasma Sheath

\section{Introduction}

Previous work by the authors [1] investigated the plasmapropellant interaction encountered in electrothermal chemical (ETC) guns. In ETC gun ignition, a plasma discharge replaces the conventional explosive ignition source. Among the enhancements encountered in ETC guns are reduced ignition delay time, enhanced burn rate, and significant increases in muzzle exit velocity [2].

In recent years there has been much experimental investigation into the plasma-propellant interaction (PPI) [3], [4], [5]. In the same time period, however, there has been very little modeling work investigating the PPI, with the most extensive ETC modeling to date investigating either the plasma source [6] or the bulk plasma [7], and not the PPI. The ETC work of the authors is focused on creating detailed models of the PPI to help determine just what physical phenomena are important in ETC ignition.

Previous modeling of ETC ignition has not looked at plasma sheath effects that should be present in the PPI. A plasma sheath would increase the ion velocity at the propellant surface, thus increasing the ion's kinetic energy. A sheath is formed at any solid boundary in contact with a plasma. The faster moving electrons hit the wall, or in this case the propellant bed, before the slower moving ions. This initial stream of electrons causes the wall to develop a

* Corresponding author; e-mail: aporwitz@umich.edu negative charge until the electrostatic potential at the wall is large enough to repel incoming electrons and balance the ion and electron fluxes. Such a condition is called a floating wall. The plasma sheath is the region over which the electrostatic potential decreases from the bulk plasma potential to the wall potential [8].

Developed here is a computational model for a collisional plasma sheath with a high density neutral background, tailored specifically for ETC application. The geometry adopted is illustrated in Figure 1. The sheath model is coupled to the thermal/ablation model previously developed [9], resulting in data on the time evolution of the plasma properties, with emphasis placed on the convective heat flux to the propellant bed.

The two propellants studied here are a double-base, JA2, and a nitramine composite, XM39. These propellants have very different optical properties. JA2 is optically semitransparent and it has been demonstrated that in-depth physical changes occur up to approximately $1 \mathrm{~mm}$ into the propellant when exposed to plasma radiation [5], [10]. XM39 is opaque and recent experiments indicate its reflectivity may be as high as $50 \%$ [11]. The majority of current theories attempting to describe the PPI contend that radiation penetration, effecting in-depth chemical changes and acting as a secondary heating mechanism, is a major cause of the enhancements one sees in ETC guns [5], [7], [10]. The present work provides additional support for the

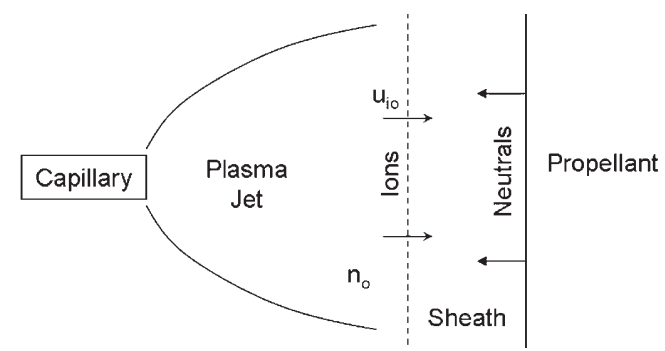

Figure 1. Diagram of the model geometry. Present model represents the region of the sheath coupled to a prior model for the propellant ablation. 
importance of radiation in the PPI, but that radiation is not necessarily the dominant heating mechanism.

In Section 2 we describe the fundamentals of the plasma sheath and the governing equations. We briefly present sheath results at fixed neutral density in Section 2.1. In Section 2.2 we explain how the previous ablation model was coupled to the present sheath model to yield the convective heat flux to the propellant bed. Results and Discussion are presented in Section 3.

\section{Sheath Model}

The one-dimensional, mono-energetic sheath model implemented here assumes Boltzmann electrons and uniform background neutral density. The capillary discharge model indicates an electron temperature of $T_{e}=1.5 \mathrm{eV}$ is appropriate [6]. The equations to be solved are ion continuity and momentum, and the Poisson equation for the electrostatic potential. By making the cold ion assumption we neglect the ion density gradient in the momentum equation and numerically integrate via the Runge-Kutta method [12], starting at the bulk plasma and proceeding toward the wall. This requires an additional equation for completeness which is given by the relation between electric field and potential Eq. (1).

For clarity of solution, the equations are non-dimensionalized. All densities are non-dimensionalized by the bulk plasma density, $n_{o}$, thus the ion density is given by $N_{i}=n_{i} / n_{o}$, and the neutral density by $N_{n}=n_{n} / n_{o}$. The potential is given as $\eta=\varphi / T_{e}$, with $T_{e}$ in Volts. Velocities use the Bohm velocity, $C_{s}$, thus $u_{i}=v_{i} / C_{s}$, where $C_{s}=\sqrt{e T_{e} / m_{i}}$ and $m_{i}$ is the average atomic mass of the propellant in question. Spatial dimension use Debye length for normalization, thus $y=x / \lambda$. Lastly, electric field is non-dimensionalized to $\varepsilon=$ $E \lambda / T_{e}$. The sheath is thus represented by the following equations.

$$
\begin{aligned}
& \frac{\partial \eta}{\partial y}=-\varepsilon \\
& \frac{\partial \varepsilon}{\partial y}=N_{i}-\exp \eta \\
& \frac{\partial N_{i}}{\partial y}=-\frac{N_{i}}{u_{i}} \frac{\partial u_{i}}{\partial y}+\frac{N_{n} N_{e}}{u_{i}} \tilde{R}_{i} \\
& \frac{\partial u_{i}}{\partial y}=\frac{\varepsilon}{u_{i}}-u_{i} \alpha \tilde{\sigma} N_{n}
\end{aligned}
$$

Where $\alpha=n_{o} \lambda^{3}$, and $\tilde{\sigma}$ is the non-dimensional momentum exchange collision cross section for the propellant, given by

$$
\tilde{\sigma}=\frac{\pi}{2 \lambda^{2}} \sum_{j} d_{j}^{2} X_{j}
$$

where the extra factor of $\pi / 2$ in the cross section forces the hydrodynamic sheath treatment to match the kinetic treatment [13]. Due to lack of knowledge of the chemical makeup of the ablated propellant during the PPI, we assume that the ions and neutrals have the same elemental makeup, being that of the ablated propellant. $d_{j}$ is the variable hard sphere diameter of elemental compound $j$ present in the propellant, and $X_{j}$ is the mass fraction of element $j$ in the propellant. Table 1 contains the mass fractions and diameters used that are taken from [14] and [15].

$\tilde{R}_{i}$ in Eq. (3) is the non-dimensional ionization rate. It was found after a series of representative cases that the effect of ionization collisions on the dynamics over the plasma sheath was negligible. For simplicity, ionization has been neglected here.

For a collisional plasma sheath the ion inflow velocity at the bulk plasma interface, $u_{i o}$, is not known [16]. The physical maximum will be at or below the Bohm velocity, $u_{i o}=1$. The exact ion inflow speed depends on the characteristics of the plasma source and the plasma-air interaction happening between that source and the sheath edge. For this reason the ion inflow speed is not known, but a reasonable range can be assumed. In the present work, values for $u_{i o}$ of $0.3,0.6$, and 1 are used to see the effect that inflow velocity has on the sheath dynamics, specifically the convective heat flux reaching the propellant bed. It should be noted that changing the inflow velocity changes the ion flux into the sheath, as $N_{i}=1$ at the sheath edge.

The propellant bed is taken to be a floating wall, with the potential at the bulk plasma interface defined as $\eta_{o}=0$. With the variable ion flux the derivation of the floating potential yields

$\eta_{w}=-\ln \left(\frac{1}{N_{i} u_{i}} \sqrt{\frac{m_{i}}{2 \pi m_{e}}}\right)$

where $m_{e}$ is the electron mass. Note that with ionization neglected, the ion flux is constant across the sheath, such that $N_{i} u_{i}=u_{i o}$. The Runge-Kutta method stops integrating when $\eta=\eta_{w}$

A non-zero initial electric field is assumed to obtain the initial potential gradient that is consistent with a sheath solution. An initial value is used for a number of representative simulations in the range $10^{-8} \leq \varepsilon_{o} \leq 10^{-4}$, with there being no change in the simulation results over this variation. Thus we know that the initial electric field is arbitrary for the solution as long as it lies within this broad range.

Table 1. Propellant composition by mass and variable hard sphere diameters used for momentum exchange collisions.

\begin{tabular}{llll}
\hline Species & $X_{j}, \mathrm{JA} 2$ & $X_{j}, \mathrm{XM} 39$ & $d_{j}\left(10^{-10} \mathrm{~m}\right)$ \\
\hline $\mathrm{H}$ & 0.02345 & 0.03646 & $2.92\left(\mathrm{H}_{2}\right)$ \\
$\mathrm{C}$ & 0.09251 & 0.2248 & $1.544^{\mathrm{a}}$ \\
$\mathrm{N}$ & 0.1955 & 0.2969 & $4.17\left(\mathrm{~N}_{2}\right)$ \\
$\mathrm{O}$ & 0.6883 & 0.4418 & $4.07\left(\mathrm{O}_{2}\right)$ \\
\hline
\end{tabular}

${ }^{a}$ Atomic diameter used for carbon as variable hard sphere value could not be found. 


\subsection{Sheath Results at Fixed Neutral Density}

Before coupling the sheath to the ablation model we shall examine some results for fixed background neutral density, $N_{n}$, as they warrant discussion. The initial conditions assume a bulk plasma in the vicinity of the sheath with a small electric field, $\varepsilon_{o}=10^{-5}$; grounded potential, $\eta=0$; no charge separation, $N_{i}=N_{e}$; and no background neutrals, $N_{n}=0$. At the beginning of the simulation the ions begin to experience a drag force due to the second term on the right hand side of Eq. (4). If this drag force is not immediately overcome by the electrostatic acceleration term in Eq. (4) then the ions slow down. Due to continuity Eq. (3) the ions begin to pile up near the interface. Since the electrons are non-collisional, eventually the charge separation is great enough that the electrostatic term in Eq. (4) overcomes the drag term and the ion density begins to drop off again.

In Figure 2 are shown representative sheath solutions for fixed neutral density. In Figure 2 a we can see a classic sheath solution with only a small rise in ion density after the inflow. Figure $2 \mathrm{~b}$ shows a typical buildup in ion density. As the neutral density is increased, the ion density peak becomes higher and thinner. Sheridan and Goree briefly mention this effect in [17], however these profiles have not been
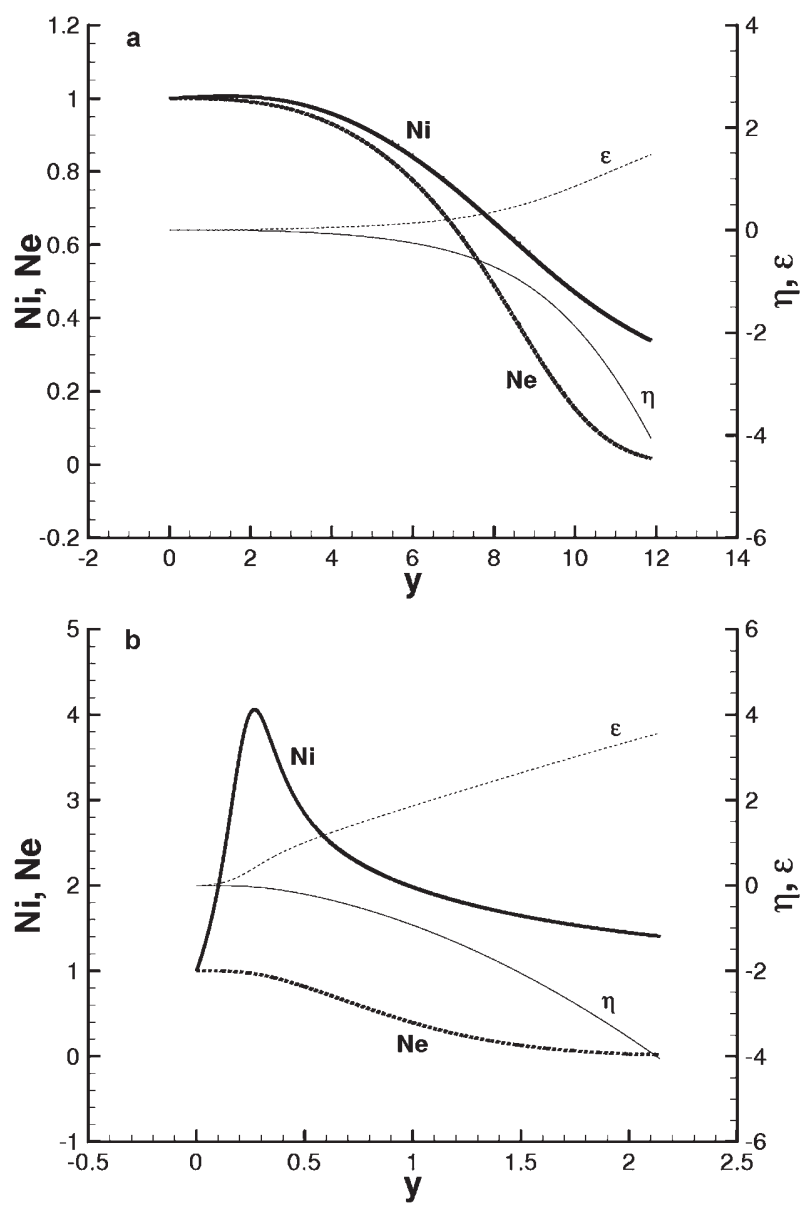

Figure 2. Sheath solutions at constant neutral density for JA2 with $u_{i o}=1, n_{o}=10^{22} \mathrm{~m}^{-3}$. (a) $N_{n}=10$ (b) $N_{n}=10^{4}$. published. The cold ion, Runge-Kutta formatted model employed here is common in sheath modeling, and these results, although physical for the conditions imposed, can be considered a limitation in this approach.

\subsection{Ablation Model Coupling}

Coupling the sheath model to the ablation model allows us to determine the sheath dynamics over the course of the plasma pulse duration, $\Delta t=280 \mu \mathrm{s}$ [1]. With the propellant surface temperature profile and a model for the vapor pressure of each propellant, the neutral density in the sheath is given as

$n_{n}=\frac{P\left(T_{s}\right)}{k T_{s}}$

where $P\left(T_{s}\right)$ is the propellant vapor pressure at surface temperature $T_{s}$, and $k$ is Boltzmann's constant. Since $T_{s}$ is a function of time, we obtain the background neutral density as a function of time during the plasma pulse. By discretizing the pulse time we are able to solve the steady state sheath equation under each neutral density condition and patch these solutions together to obtain the time evolution of the sheath. Implicit in this assumption is that the formation time of the sheath is much less than the discretization of the pulse duration. The formation time of the sheath can be estimated as $\lambda / v_{i}$, which is on the order of $10^{-11}$ seconds for the cases shown here. Since the pulse discretization is $2.80 \times 10^{-5}$ seconds, the assumption is valid.

The previous work by the authors resulted in an ablation model for use in modeling the PPI. Combined with a set of experimental results it was possible to determine an effective constant total heat flux to the propellant bed needed to replicate a measured ablated mass loss [1]. The total heat flux to the bed, $q_{\text {total }}$, was determined as a function of bulk plasma density for the same experimentally determined ablated mass, as the plasma density in the experiment was not known. A range of possible bulk plasma densities was determined via a capillary discharge model [6], that range was used in the previous ablation model and here as well.

We calculated the convective heat flux, $q_{\text {conv }}$, as a function of time during the plasma pulse, and at a range of bulk plasma densities. This was done in an attempt to determine the relative importance of radiation in the PPI under the assumption that the total heat flux can be decomposed as $q_{\text {total }}=q_{\text {conv }}+q_{\text {rad }}$, where $q_{\text {rad }}$ is the unknown effective surface radiative heat flux from the plasma to the propellant bed. The convective heat flux to the propellant bed, under the assumption of no ionization in the sheath, is

$q_{\text {conv }}=\left(n_{o} C_{s} u_{i o}\right)\left(\frac{m_{i}}{2}\left(C_{s} u_{i w}\right)^{2}+2 e T_{e}-e T_{e} \eta_{w}\right)$

where $u_{i w}$ is the ion-wall impact velocity [18]. The first term in Eq. (7) is the kinetic energy of the ions impacting the wall. 
The second and third terms represent the electron impact energy, with the former being the kinetic energy of Maxwellian electrons, and the latter an energy bias, as only electrons that can overcome the potential hill of the sheath can strike the wall.

\section{Results and Discussion}

We show a representative plot of the convective heat flux versus ion inflow velocity at a range of constant background neutral densities for JA2 in Figure 3. Clearly there is a correlation between neutral density and convective heat flux; as neutral density increases, the convective heat flux decreases. This has a large impact when coupled with the ablation model, since as time progresses the surface temperature increases, which increases the vapor pressure and thus the neutral density. This can clearly be seen for the representative case presented in Figure 4, which shows the propellant surface temperature and associated neutral density for JA2 taken from the previous work [1], along with the model results for convective heat flux versus time over the course of the plasma pulse.

The corresponding plots for XM39 are shown in Figure 5. Note that XM39 exhibits a higher convective heat flux than does JA2. We can clearly see a difference in curvature in convective heat flux for XM39 compared with JA2 at early times. This is easily explained by examining the neutral density near the start of the pulse. The initial neutral density is on the order of $N_{n}=0.1$ for XM39, indicating that the plateau approached on the left hand side of Figure $5 \mathrm{~b}$ is actually the collisionless sheath limit. We expect the level plane reached on the right to be the fully collisional sheath limit.

Examining Eq. (7), we see that all variables are defined analytically, such as $\eta_{w}$, or found from prior work, such as $N_{n}$. The only unknown that the simulation must solve for is $u_{i w}$, the ion-wall impact velocity. Analytical solutions exist for the ion-wall impact velocity in the extremes of collisionless

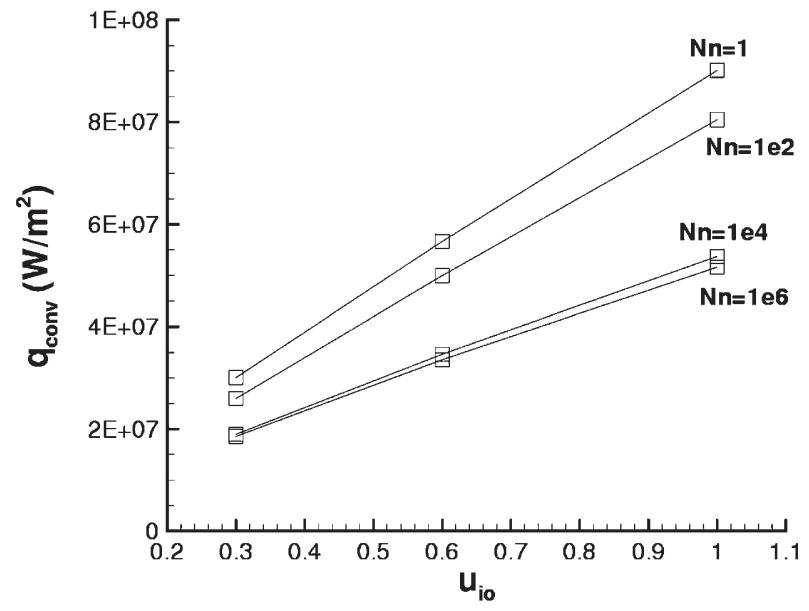

Figure 3. Representative convective heat flux versus ion inflow velocity at fixed neutral density for JA2 at $n_{o}=10^{22} \mathrm{~m}^{-3}$. a
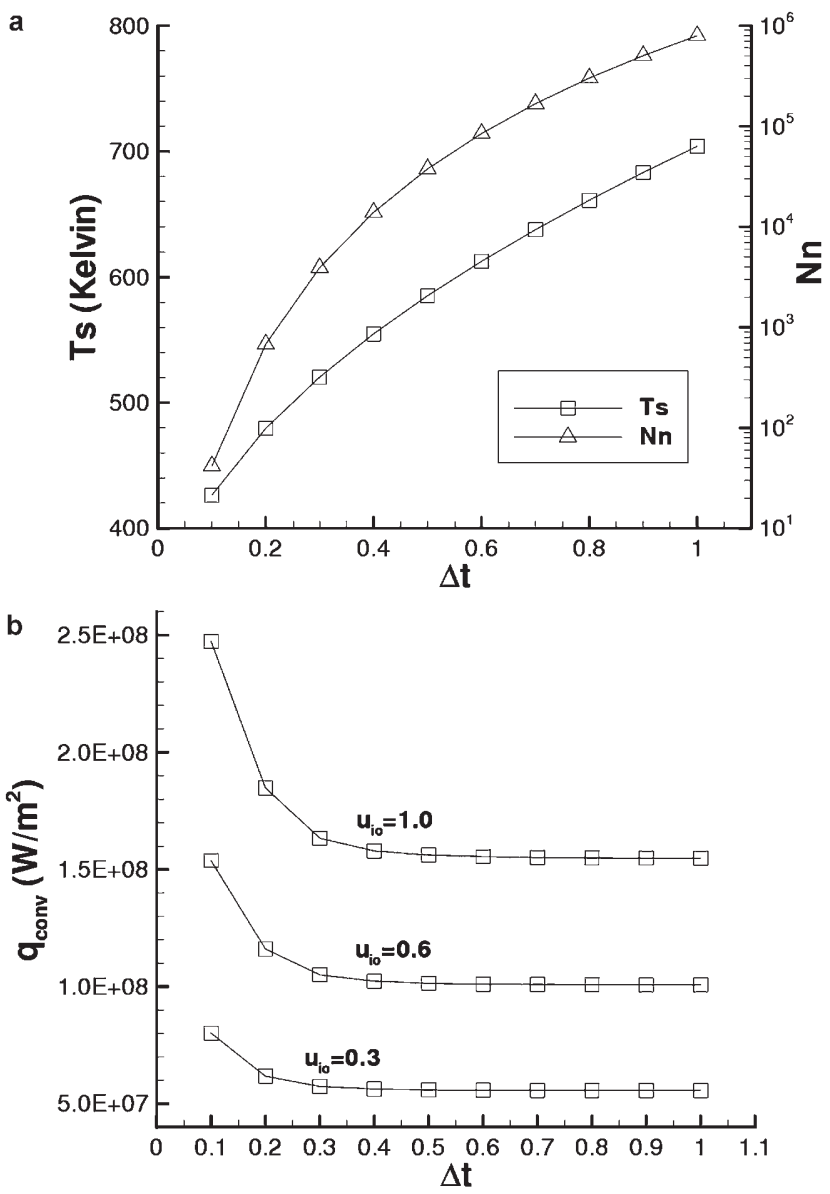

Figure 4. JA2 at $n_{o}=3 \times 10^{22} \mathrm{~m}^{-3}$. Total heat flux was $3.74 \times$ $10^{8} \mathrm{~W} / \mathrm{m}^{2}$. (a) Propellant surface temperature and neutral density during plasma pulse. (b) Convective heat flux for different ion inflow velocities as a function of time.

and fully collisional sheaths [17]. With these values known, we can simply substitute in the analytical solutions into Eq. (7) and calculate the convective heat flux for these two limiting cases at each time step. In the fully collisional sheath limit, the ion-wall impact velocity is given by

$u_{i w}=\left(\frac{5}{2} \frac{-\eta_{w} u_{i o}}{\left(\alpha N_{n} \tilde{\sigma}\right)^{2}}\right)^{1 / 5}$

For the collisionless limit the result is more complicated, yielding

$u_{i w}=\left[2\left(\frac{u_{i o}^{2}}{2}-\eta_{w}\right)\left(1-\frac{4 \sqrt[4]{2}}{7} \alpha N_{n} \tilde{\sigma} \frac{\left(-\eta_{w}\right)^{3 / 4}}{\sqrt{u_{i o}}}\right)\right]^{1 / 2}$,

which is a Child's Law sheath [17]. These asymptotic values are compared to the simulation results and are found to yield excellent agreement for the highly collisional end of the heat flux, indicating that toward the end of the plasma pulse the sheath belongs to the fully collisional regime. For most cases the sheath is never collisionless, as the non-dimensional 
a
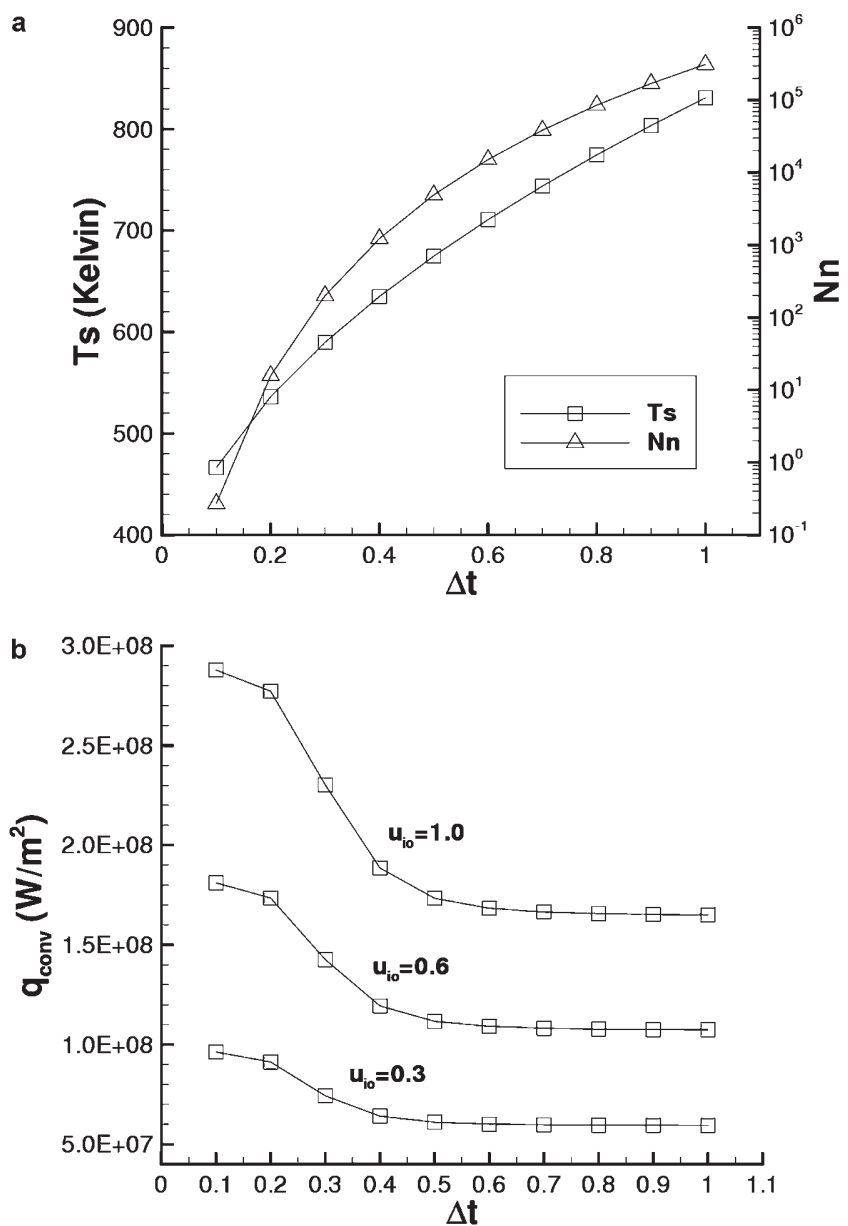

Figure 5. XM39 at $n_{o}=3 \times 10^{22} \mathrm{~m}^{-3}$. Total heat flux was $2.67 \times$ $10^{8} \mathrm{~W} / \mathrm{m}^{2}$. (a) Propellant surface temperature and neutral density during plasma pulse. (b) Convective heat flux for different ion inflow velocities as a function of time.

neutral density is generally greater than one at all time steps. The sheath is in a transition regime, neither collisionless nor fully collisional, for a significant fraction of the plasma pulse for all data sets.

One of the best cases for illustration is shown in Figure 6, which is the $u_{i o}=1$ case from Figure 5b. At $\Delta t=0.1$ we can see that the sheath is nearly collisionless. At $\Delta t=0.2$ the simulation result has deviated from the collisionless solution, indicating that the sheath has entered a transition regime that continues until $\Delta t=0.6$. The simulation makes a smooth transition from near collisionless to fully collisional.

We present a time average convective heat flux over the course of the plasma pulse for comparison to the total heat flux obtained from [1], which assumed a constant heat flux during the plasma discharge. The results of this calculation are shown in Figure 7 for both propellants. The range of bulk plasma density is chosen to yield convective heat fluxes approximately equal to, or lower than, the total heat flux as calculated in [1]. It should be noted that $n_{o}=10^{22} \mathrm{~m}^{-3}$ is the middle ground of our estimate of possible plasma densities generated in the experimental chamber by the capillary plasma source [1], [6].

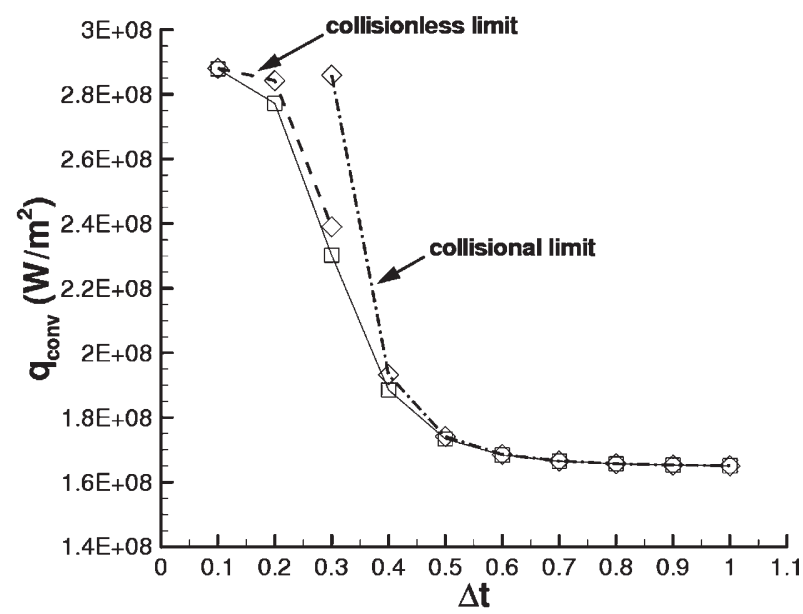

Figure 6. XM39 at $n_{o}=3 \times 10^{22} \mathrm{~m}^{-3}, u_{i o}=1$. A representative simulation result as compared to the convective heat fluxes for a collisionless sheath and a fully collisional sheath.

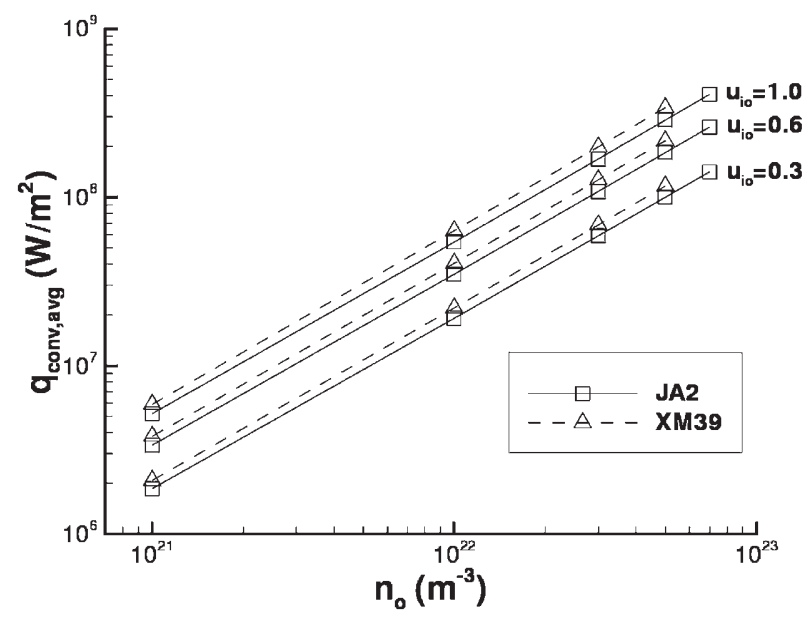

Figure 7. Average convective heat flux for each propellant as a function of bulk plasma density.

Comparing the two propellant's convective heat fluxes, while keeping in mind their optical properties, yields much insight. For a given combination of bulk plasma density and ion inflow velocity, XM39 has a higher convective heat flux than JA2, as illustrated by Figure 7 . However, for a given bulk plasma density, JA2 has a higher total heat flux than XM39 [1]. Since JA2 is susceptible to plasma radiation, and XM39 is not, we expect JA2 to have a higher radiative heat flux. The data presented here indicates that the importance of radiation is even more pronounced. Previously the authors denoted the difference between the total heat flux of JA2 and XM39 as $\Delta q$, and postulated that if JA2 and XM39 had the same convective heat flux then $\Delta q$ represented the effect that radiation, both penetrating and surface heating, had on JA2 [1]. This new data modifies that conclusion, since it is clear that XM39 has a higher convective heat flux. JA2 has more ground to make up on XM39 to match and surpass its total heat flux. 
We show the fraction of the total heat flux that is accounted for by the average convective heat flux across a sample of bulk plasma densities in Figure 8. Since the bulk plasma density in the original experiment is not known, we can not say at present which percentage is the correct one. Future work will attempt to determine the plasma density at the sheath edge by means of a plasma jet flow simulation.

This study raises the possibility that radiation is not necessarily the dominant heat transport mechanism in the PPI, as a significant portion of the total heat flux can be accounted for by convective heating due to plasma particle flux to the propellant bed. One reason for thinking that plasma radiation is the dominant heat source in the PPI is the fact that the radiative flux available from the plasma can more than account for the total heat flux needed for ignition. For a blackbody plasma, $q_{\text {rad }}=\sigma_{s} T_{e}^{4}$, where $\sigma_{s}$ is the StephanBoltzmann constant [19]. For $T_{e}=1.5 \mathrm{eV}$ this yields an available radiative flux of $q_{\text {rad }}=5.2 \times 10^{9} \mathrm{~W} / \mathrm{m}^{2}$. However, there is evidence that the actual radiative flux to an ETC propellant is much lower [20]. The present study yields additional support to the idea that some degree of radiation shielding is occurring during the PPI.
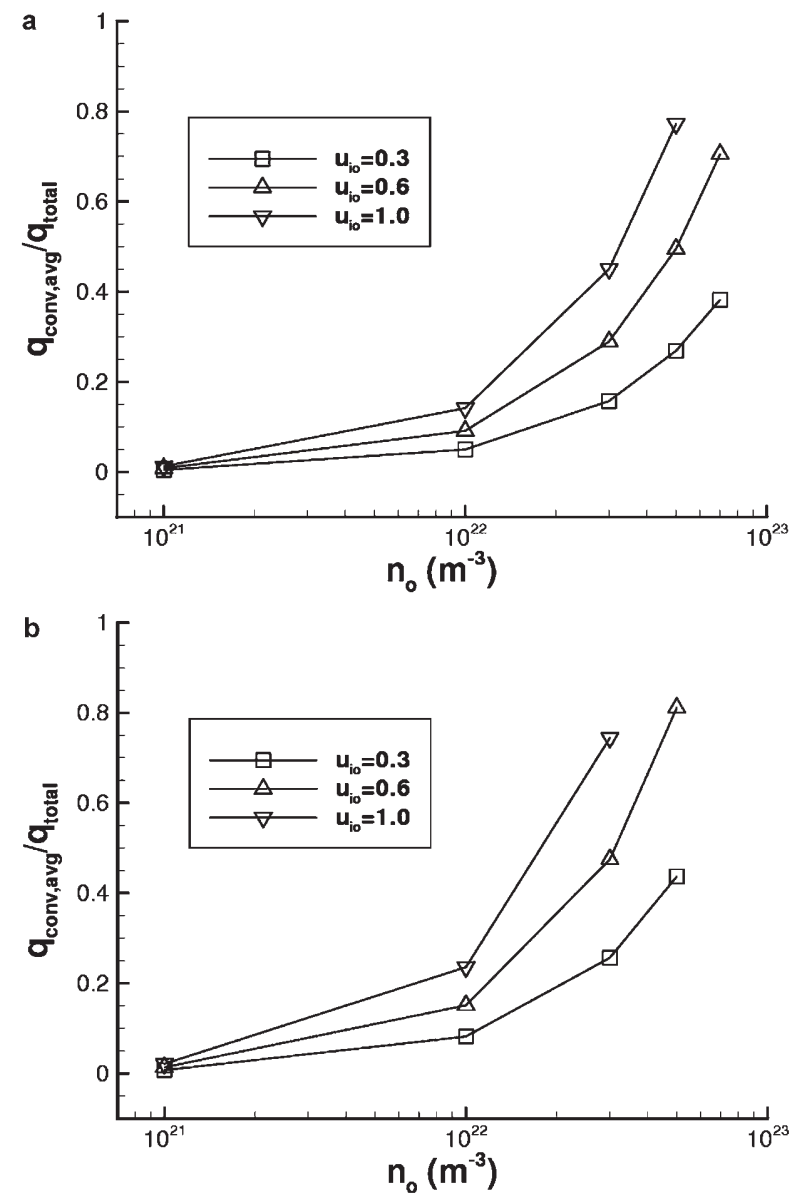

Figure 8. Fraction of the total heat flux that is accounted for by the average convective heat flux at different plasma densities and ion inflow velocities. (a) JA2 (b) XM39.
It has been proposed [21] that recombination reactions affecting species liberated by the PPI (plasma and propellant radicals) at or near the propellant surface may be a significant source of heat flux to the propellant bed. We do not rule out this possibility, and it is likely that such effects are contained within the heat flux labeled $q_{r a d}$, since this was found by subtracting out the convective heat flux from the total. However, given the evidence, we contend that the dominant factors in the PPI are radiation and convective heat fluxes, with the order of dominance determined mainly by the propellant choice. Additional heat sources, which are chemical in nature, can include the aforementioned recombination reactions, heat sources due to film deposition on the propellant surface, as well as any effects due to in-depth chemical changes caused by penetrating plasma radiation. These chemical effects have not been thoroughly explored in modeling the PPI. Future work will attempt to model these chemical effects not only as a heat source, but as a factor affecting the ablation rate of the propellant.

\section{Conclusion}

A coupled plasma sheath/ablation model was developed for electrothermal chemical (ETC) gun applications. The model finds that XM39, an optically opaque propellant, has a higher convective heat flux than JA2, which is semitransparent. Since previous modeling indicates that JA2 has a higher total heat flux than XM39, we conclude that plasma radiation is an important heat source in the plasmapropellant interaction, but that the convective heat flux can represent a major contribution to the total heat flux. This new data reaffirms previous conclusions from modeling work that when picking a propellant for ETC application, the optical properties can not be ignored, and that the best choice for ETC gun propellant is one which allows plasma radiation to penetrate its interior. Results clearly show that the sheath begins in the near collisionless regime, undergoing a smooth transition to the fully collisional regime before the end of the plasma pulse. Although this study indicates that having a low vapor pressure would increase the convective heating of the propellant, we know that this would also result in a lower ablation rate, which is a negative effect to ETC ignition overall. It is also unknown how much radiation shielding is occurring due to ablated propellant neutrals, and perhaps there is an optimum balance between ablation and radiation shielding to yield the ideal vapor pressure for ETC application.

\section{References}

[1] A. J. Porwitzky, M. Keidar, I. D. Boyd, Modeling of the Plasma-Propellant Interaction, IEEE Trans. on Magnetics 2007, 43, 313.

[2] Th. H. G. G. Weise, P. Schaffers, O. Stark, L. P. Svanberg, L. Gustavsson, J. Dahlberg, ETC-Investigations in the Upper Medium Calibre Range, 13th Electromagnetic Launch Symposium, Potsdam, Germany, 22-25 May 2006, 2006. 
[3] A. W. Williams, K. J. White, Plasma-Propellant Interactions Studies: Measurements of Heat Flux Produced by Hydrocarbon Ablation-Supported Plasmas, IEEE Trans. on Magnetics 2004, 37, 1.

[4] J. Li, T. A. Litzinger, S. T. Thynell, Interaction of Capillary Plasma with Double-Base and Composite Propellants, $J$. Propulsion Power 2004, 20, 4.

[5] R. A. Pesce-Rodriguez, R. A. Beyer, A Theory of PlasmaPropellant Interactions, 2004, ARL-TR-3286.

[6] M. Keidar, I. D. Boyd, Ablation Study in the Capillary Discharge of an Electrothermal Gun, J. Appl. Phys. 2006, 99, 053301.

[7] K. Kappen, U. H. Bauder, Simulation of Plasma Radiation in Electrothermal-Chemical Accelerators, IEEE Trans. on Magnetics 1999, 35, 1.

[8] M. A. Lieberman, A. J. Lichtenberg, Principles of Plasma Discharges and Materials Processing, Wiley-Interscience, Hoboken 2005, p. 172.

[9] M. Keidar, I. D. Boyd, I. I. Beilis, On the Model of Teflon Ablation in an Ablation-Controlled Discharge, J. Phys. D: Appl. Phys. 2001, 34, 1675.

[10] R. A. Beyer, R. A. Pesce-Rodriguez, The Response of Propellants to Plasma Radiation, 39th JANNAF Combustion Meeting, Colorado Springs, 1-5 December 2003.

[11] R. Beyer, Army Research Lab, Aberdeen Maryland, private communication, 2005.

[12] C. F. Gerald, P. O. Wheatley, Applied Numerical Analysis, Addison-Wesley Publishing Company, Boston 2004, p. 342.

[13] Z. Sternovsky, S. Robertson, Numerical Solutions to the Weakly Collisional Plasma and Sheath in the Fluid Approach and the Reduction of the Ion Current to the Wall, IEEE Trans. on Plasma Science 2006, 34, 3.

[14] M. Miller, Thermophysical Properties of Six Solid Gun Propellants, 1997, ARL-TR-1322.
[15] G. A. Bird, Molecular Gas Dynamics and the Direct Simulation of Gas Flows, Oxford University Press, New York, 1994, Appendix A.

[16] M. Keidar, I. I. Beilis, Transition From Plasma to SpaceCharge Sheath Near the Electrode in Electrical Discharges, IEEE Trans. on Plasma Science 2000, 28, 2.

[17] T. E. Sheridan, J. Goree, Collisional Plasma Sheath Model, Phys. Fluids B 1991, 3, 10.

[18] M. Keidar, I. D. Boyd, I. I. Beilis, Electrical Discharge in the Teflon Cavity of a Coaxial Pulse Plasma Thruster, IEEE Trans. on Plasma Science 2000, 28, 2.

[19] B. W. Carroll, D. A. Ostlie, An Introduction to Modern Astrophysics, Addison-Wesley Publishing Company, New York 1996, p. 77.

[20] M. Das, S. T. Thynell, J. Li, T. A. Litzinger, Transient Radiative Heat Transfer from a Plasma Produced by a Capillary Discharge, 40th JANNAF Combustion Meeting, Carleston SC, 13-17 June 2005.

[21] M. J. Taylor, Measurement of Organic Plasma Temperature for $155 \mathrm{~mm}$ ETC Gun Ignition, 13th Electromagnetic Launch Symposium, Potsdam, Germany, 22-25 May 2006.

\section{Acknowledgements}

The authors gratefully acknowledge the financial support by the Army Research Office, Grant No. W911NF-04-1-0251 (Dr. Kevin McNesby, technical monitor).

(Received October 19, 2006; Ms 2006/044) 